\title{
Coordinating the Global Information Grid Initiative with the NG9-1-1 Initiative
}

\section{IEEE International Conference on Technologies for Homeland Security}

\section{Michael Schmitt}

May 2008

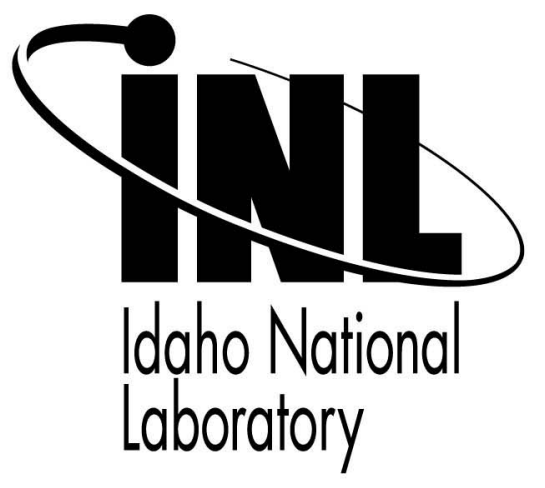

This is a preprint of a paper intended for publication in a journal or proceedings. Since changes may be made before publication, this preprint should not be cited or reproduced without permission of the author. This document was prepared as an account of work sponsored by an agency of the United States Government. Neither the United States Government nor any agency thereof, or any of their employees, makes any warranty, expressed or implied, or assumes any legal liability or responsibility for any third party's use, or the results of such use, of any information, apparatus, product or process disclosed in this report, or represents that its use by such third party would not infringe privately owned rights. The views expressed in this paper are not necessarily those of the United States Government or the sponsoring agency. 


\title{
Coordinating the Global Information Grid Initiative with the NG9-1-1 Initiative
}

\author{
Michael Schmitt, Idaho National Laboratory, Idaho Falls, Idaho, 208-526-9556, michael.schmitt@inl.gov
}

\begin{abstract}
As the Department of Defense develops the Global Information Grid, the Department of Transportation develops the Next Generation 9-1-1 system. Close examinations of these initiatives show that the two are similar in architectures, applications, and communications interoperability. These similarities are extracted from the lowest user level to the highest commander rank that will be involved in each network. Once the similarities are brought into perspective, efforts should be made to collaborate between the two departments.
\end{abstract}

\section{INTRODUCTION}

On April 6, 2007, the Department of Transportation (DoT) published the "Next Generation 9-1-1 (NG9-1-1) System Initiative Concept of Operations." This document provided first responders and equipment vendors valuable insight into the goals that the NG9-1-1 initiative set out to accomplish. A short time later, the DoT published the "System Description and High-Level Requirements Document," which provided a further detail to the architecture of NG9-1-1. In both of these documents, the mission statement is to enable public access to 9-1-1 through virtually any device. [1], [2] This capability is not available through the current 9-1-1 system. Additionally, both documents require that first responders have interoperable communications and access to required information.

Around the same time, the Department of Defense (DoD) published the "Global Information Grid (GIG) Architectural Vision." This document serves as the blueprint for the DoD, as well as other agencies, on how a net centric force will be implemented and deployed. The stated GIG goal is to "ensure timely and trusted information is available where it is needed, when it is needed, and to those who need it."[3] This document goes on to conceptualize the architecture, connectivity, and applications that the war fighter will use to achieve the stated goals.

Studies conducted at Idaho National Laboratory (INL) have discovered striking similarities between the DoD and DoT initiatives. For example, while DoD uses the term "Net-Centric" to describe the GIG initiative, the first responder community uses the term "Interoperability" to describe the NG9-1-1 initiative. In the context of their respective architectural visions, these terms are in fact interchangeable.
The military requirements for a net centric force are to connect disparate communications systems to a network and allow effective access to information from anywhere within the network. On the other hand, the first responder community requires that many disparate communications systems network with other disparate systems to receive information.

The similarities of the NG9-1-1 system and the GIG system begin with the high-level architectures that both propose. As discussed in Section II, both architectures call for a flow down of information from multiple sources to multiple users. Additionally, the architectures ensure that there is some form of interoperability between disparate systems that may be attached to the network. Finally, the architectures are built to be survivable. In the case of the NG9-1-1 model, there are provisions for operational overflow to different Public Safety Answering Points (PSAP), either collocated or geographically separated. For the GIG, survivability is accomplished by having multiple systems or sources operate in parallel so that if one system or source is disabled, information can still flow to the end user.

Another aspect of the two initiatives that is fundamentally similar is the applications that they will use, which are discussed further in Section III. Both the NG9-1-1 and GIG concepts require the ability to transfer voice, data, and video. The requirements ensure that applications can be used on demand, with as little delay as possible. Although the data may be applied in different ways, fundamentally the applications are very similar. For example, the Emergency Operations Center may require streaming video of a fire to determine resource requirements. On the same level, the on-site military commander may require streaming video of a firefight to initiate tactical plans.

Connectivity is perhaps the most arduous task both departments face when deploying their respective networks. It is logistically impractical and extremely costly for DoT to expect all agencies to adopt end user equipment that meets a specific standard. The same can be said of the DoD initiative. Therefore, instead of requiring hundreds of thousands of end users to adapt to a new network, both architectures require the networks to be flexible enough to adapt to the end user. Section IV discusses the different ways each initiative will achieve interoperability.

Finally, Section V draws the conclusion that there is too much at stake in both of these initiatives to ignore the 
chance for collaboration between the DoD and DoT. There is a common thread that each entity can leverage, and this is the Department of Homeland Security (DHS). As an agency that coordinates much of the military's mission of civil support, works with the national guard and has an interest in first responder communications, DHS has a stake in both initiatives.

\section{Architecture}

The first step when comparing the NG9-1-1 initiative with the GIG initiative is to examine the architectures of both systems. NG9-1-1 utilizes what is called the "Community Model" as the baseline for all of the low-level internal architectures that comprise the NG9-1-1 system, which is
Both architecture models show the continuity concepts of operations, multiple access, and geographical reach.

\section{NG9-1-1}

The key concept in the Community model is that information can flow to and from multiple sources, eliminating bottlenecks while streamlining operations. This model addresses the future technology needs required by first responders by ensuring the implementation of the systems is flexible enough to adapt to those needs.

\section{NG9-1-1 PSAP Decentralization}

New to the emergency communications arena is the

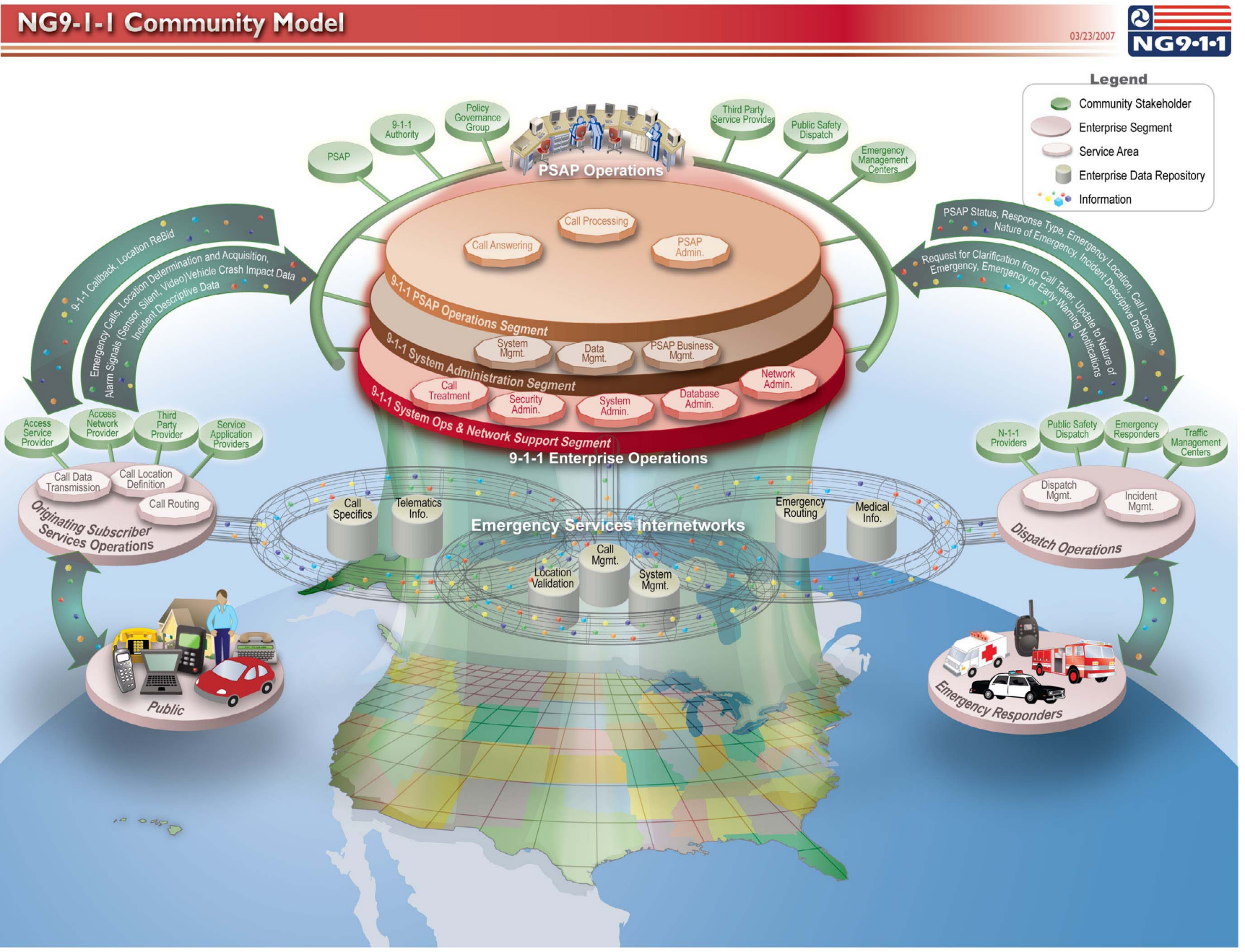

Figure 1 - NG9-1-1 Community Model [1]

shown in Figure 1. The GIG architecture depicts a model that connects all of the different users and networks. decentralization of the PSAPs. By doing this, NG9-1-1 shows that geographic location does not dictate which PSAP will be tasked during an emergency, although it is 
likely that small-scale events will be handled at the local PSAP. Should there be a large-scale event, there can be overflow to PSAPs that are not affected by the event. For example, if an earthquake strikes California and the local PSAPs are disabled, theoretically, a less-tasked PSAP in Alabama could help relieve some of the load from the area.

\section{NG9-1-1 Using Internet Protocol}

Delving further into the recommended architecture reveals a large dependency on IP-capable equipment. Most modern networks rely on IP communications to reduce bandwidth while increasing throughput and the NG9-1-1 system is no different. The architecture documentation relies on IP communications to transport radio communications, database applications, and interPSAP operations, most likely the IPv6 standard.

\section{Global Information Grid}

The GIG is designed so that all of the different entities are indicating a never-ending cycle, which is shown in Figure 2. Ideally, the architecture relies on a System of Systems concept. This is where there are many different systems integrated together to form the network. The System of Systems is important to the network architecture, since this type of implementation typically does not allow for a single point of failure network wide. Instead, if there is a failure, other portions of the network will not be affected by the outage. In some instances, other parts of the network may be able to provide back up services, causing minimal interruptions. This is the foundation to the survivability of the network.

\section{Global Information Grid Quality of Service}

A major hurdle for the GIG is to implement a Quality of Service (QoS) mechanism that is robust enough to handle the many diverse applications that will cross the network. The GIG architecture requires that all users, even those at the edge of the network, have sufficient bandwidth to send and receive the information that they need. This

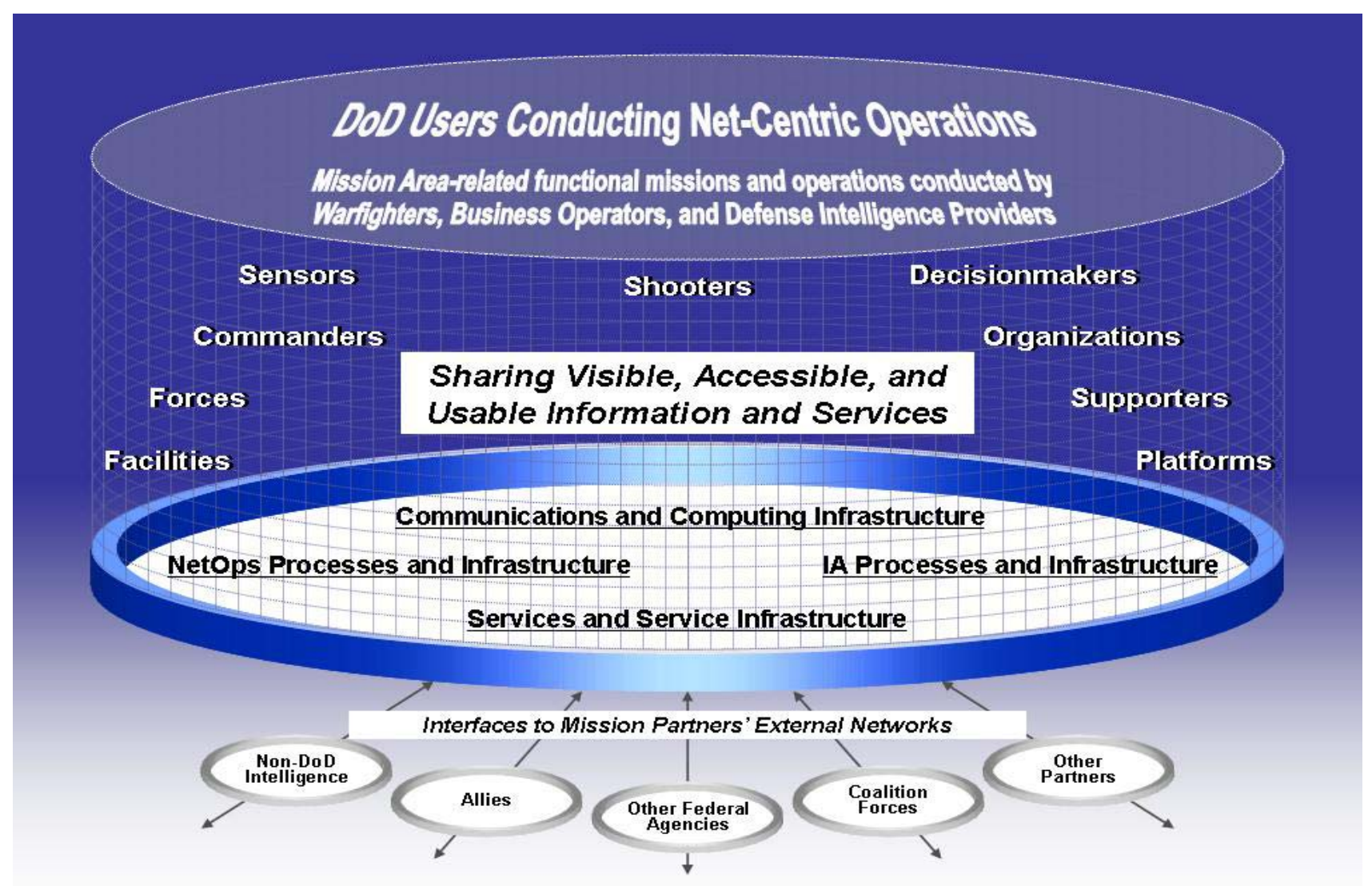

Figure 2 -- GIG Architectural Vision

able to send and receive information from multiple sources. The architecture allows any type of radio system to access the network and share information. Since the GIG is expected to be global, the depiction is circular, means that the system will have to be designed with a good QoS mechanism. In addition to requiring enough bandwidth, the data is also expected to be seen in near 
real time in almost all instances. Again, this lends itself to a robust QoS mechanism.

\section{Global Information Grid Security}

As with any military communications system, security is of the highest importance, and this holds true for the GIG. The architecture needs to be able to handle all types of classified and unclassified data. This means routing to ensure that classified information goes to the intended receivers as well as decrypting classified information from disparate sources. The requirement adds to the complexity of the system architecture by making the security rigid enough to remain secure, but flexible enough to adapt to the different security methods deployed.

\section{Similarities between Architectures}

As can be seen in the NG9-1-1 and the GIG conceptual diagrams, the similarities are striking. Both architectures require the systems to allow for geographically separated operations, although one is global while the other is confined to the United States. Both architectures require a highly reliable QoS Mechanism to ensure that information is available to users regardless of the location. Finally, both architectures call for a system of systems integration.

For both architectures, the first priority is in the system of systems integration. Integrating multiple systems from many vendors is a challenge. There will be standards developed to ensure compatibility between all of the pieces. This is one of the areas where the DoD and DoT can collaborate. By working together on this issue, the departments can develop a common standard that is applicable to all communities. This benefits the industry by reducing configurations, which reduces cost.

The second priority for both networks is the QoS implementation. There are many different implementations of QoS across cellular and data networks, some better than others. By using the best practices from industry and adapting them to fit the needs of the first responder and war fighter, the networks will achieve the desire to provide reliable communications to all users.

Finally, security is an implementation issue with both networks. While this is not necessarily a high priority for the NG9-1-1 architecture, this is an extremely high priority for the GIG architecture. Since there are many different levels of security required for the GIG, the implementation will be much more complex than in the NG9-1-1 implementation, where security will be straightforward. It is for this reason that the NG9-1-1 planners should cooperate with the DoD security personnel to determine the GIG implementation and possibly distill it down to what the requirements for NG91-1 will be.

\section{Applications}

Applications used by first responders and the military services are similar, if not in scale then certainly in urgency. Typically, applications are divided into three core groups: Voice, Video, and Data.

\section{Voice Services}

Voice services were once considered the highest priority, and they still should be. Voice is the most common method to relay information, as well as being the cheapest. In the NG9-1-1 system, voice service will be used at all points of the transaction from the person needing assistance, to the PSAP operator, and finally to the first responder. In the GIG system, the role voice plays is equally important. The soldier on the ground must be able to talk to headquarters, who then speak to the next line of command, and so forth. For both initiatives, voice service is still the foundation for all information exchanges.

The similarity of the importance of voice service in the GIG and NG9-1-1 initiatives is striking. In each initiative, the lowest user level is expected to have access to some voice service. Depending on which initiative is referred to, this is either the soldier or the first responder. In terms of voice applications, the soldier and first responder can be directly compared since presumably both are on life-threatening missions, both need survivable voice connections, and both need information in real time.

\section{Streaming Video Services}

Streaming video service is relatively new to the military and first responder communities. The military will use streaming video to monitor battle conditions, real-time damage assessments, and surveillance. The first responder community will use streaming video for criminal activity, such as high-speed chases, and remote sensing for events such as collapsed buildings and surveillance.

There is a direct comparison to all of the aforementioned situations that data will be used in the different networks. For example, a military commander may want to watch, real time, how a firefight is progressing before he/she sends in additional resources. Similarly, a police commander may want to watch a high-speed chase unfold in real time before he/she assigns additional resources.

Although the streaming video applications may be deployed for different uses, fundamentally they are the same. Both initiatives call for some quality of service mechanism to allocate bandwidth properly for streaming video. Additionally, the GIG and NG9-1-1 require minimal delay time for all streaming video. With collaboration between the DoT and DoD, these requirements can be met efficiently. 


\section{Data Services}

Data service is perhaps the most complex application that needs to be addressed in both initiatives. Traditional data services will be used similarly by NG9-1-1 and GIG for situational awareness, mission planning, and mission execution. Data services are equally important to both networks for the same reasons as the other data applications, which is for life-saving information.

In the NG9-1-1 initiative, the data services start at the caller, flow up to the PSAP or to the Emergency Operations Center (EOC), and then to the first responder. This data will naturally be parsed along the way to shape it into the type of information each entity needs to perform their assigned functions. For example, the NG91-1 will allow a caller to send a text message to the PSAP. The PSAP operator will use the information in the text message to locate the caller, notify the appropriate responder, and possibly send a reply text back to the caller. The PSAP operator will then use a combination of voice/data service to alert the first responder. Voice service will be used for notification, while some data from the text, such as a location, can be sent to the onboard computer to display a map of the emergency location.

In the GIG architecture, the same flow down of data services is expected. The data can be anything, including text, files, images, or targeting data. The GIG sets the expectation that the lowest level of ground soldier, as long as the equipment is available, should be able to send and receive data. This data should then be accessible by anyone, anywhere, anytime. An example would be if a ground soldier finds a weapons bunker, but cannot identify the type of weapons. He will be able to send a picture in real-time through the GIG. It can then reach the weapons analyst who identifies the weapons. The identity is sent back to the ground troop who can take appropriate action. At the same time, the images are sent to a bomb disposal unit who has good intelligence and will be better able to respond to the threat.

Not only is there traditional data service that needs to be accounted for, there will also be Voice over IP (VoIP). VoIP is considered data since it is sent as data packets, and therefore travels over the data transport network. VoIP is extremely sensitive to network latency and thus both NG9-1-1 and GIG will have to deploy a very robust quality of service mechanism. Both initiatives have provisions for VoIP within their networks. In the NG9-11 networks, it is expected that most of the VoIP calls will be originated by a caller needing assistance. In the GIG initiative, it is expected that VoIP can be used from forward operating bases, remote locations, or even from command centers. Basically, the GIG sets the expectation to anticipate VoIP from anywhere.

\section{Applications Similarities}

When comparing all of the applications and services that the NG9-1-1 and GIG initiatives strive to offer, it is prudent to look at the lowest common denominator. In purest form, the 9-1-1 caller should be compared to the foot soldier in a life-threatening situation. Both require a clear voice link, whether it is VoIP or legacy voice service to convey their respective messages. Alternatively, the same can be said at the higher levels of the organization. A combat commander wants to see pictures of combat damage in a similar format to the EOC commander's view of damage from a natural disaster so that they both can formulate strategic and tactical plans.

Although the applications that the GIG and NG9-1-1 initiative wish to deploy will be used on different scales, the urgency and importance are very similar in most aspects. The environments for which they will be used are alike since they will be used with the same urgency regardless of the circumstance. It is for this reason that DoD and DoT should work together to develop the requirements for application interfaces.

\section{COMMUNiCATIONS INTEROPERABILITY}

Up to this point, network operability has been discussed along with the applications that each initiative envisions deploying. The remaining issue is how to create an environment where anyone, with any radio frequency and waveform, is able to connect to the network. In other words, how does one piece of the network become interoperable with another part of the network? First responders have been grappling with this problem for years, and this is not a new problem for the military either.

For first responders, the simplest form of interoperability typically means using different radios on different frequencies to communicate with each other. The impact of this issue is felt everyday by first responders everywhere. Currently, most municipalities use several different frequencies for fire, police, medical, and sheriff's departments, making it difficult for the services to communicate between each other. In large metropolitan areas, this can mean carrying several radios, which is impractical. The NG9-1-1 initiative plans to solve this problem in a couple of ways. The first solution is to create a common network over the $700 \mathrm{MHz}$ spectrum. This solution is several years off since the spectrum has yet to be allocated. The second solution is immediate and just as effective, which is to install radio translators to take the received voice, convert it to a different frequency, and transmit it. By doing this, the police department can talk directly to the fire department and so forth. Currently, several National Guard units perform this function by deploying the Joint Incident Communications Capability (JISCC) during disaster events. NG9-1-1 strives to improve on this implementation in a permanent form. 
For NG9-1-1, interoperability will grow to a bigger issue as different devices for data applications are used. Data from a police unit may need to be sent to a fire engine. Although the data is common, the devices on which they are displayed may be different, requiring different interfaces at the end device. The NG9-1-1 commission plans to incorporate standards so all devices can display data in the same form, regardless of the manufacturer.

The military definition of interoperability is similar, except on a much larger scale. Whereas the NG9-1-1 initiative needs to take into account multiple local, state, and federal agencies, the GIG needs to be capable of accepting the same types of information, but also the data coming from international users and from multiple types of secure communications. However, at the root of the military's interoperability issues are all of the different types of radios that are deployed across the various branches of the military. It is impractical to deploy new radio systems across all of the services; so many legacy forms of communication will still be used.

In order to solve the issues associated with the different frequencies and waveforms, the GIG intends to implement several techniques. The first approach is to deploy relay centers that have the capability to convert and disseminate the information across many different systems. Such a platform is likely to be an aircraft, such as CABLE. The heart of this is similar to NG9-1-1, but on a much larger scale. The second technique is to deploy Software Defined Radios (SDR). Software Defined Radios are one of the key advances in communications that will benefit both the first responder and the military operator. The SDR can adapt to most frequencies and waveforms, making it an extremely useful tool.

Finally, like NG9-1-1, the GIG has to take into account the data that will flow through the network from several different devices. The GIG must assure that data is seen similarly on different types of devices throughout the services. This can be done through standardization among all of the different equipment providers.

The fundamental needs for interoperability are the same between the GIG and NG9-1-1 initiatives, and much of the technology is similar enough to warrant a comparison of planned equipment. Standards for interfaces should be developed in conjunction with each initiative to ease the implementation and improve the interoperability of the systems.

\section{Conclusions}

In today's world of resource utilization, business and operations streamlining, and technology growth, it is in the best interest of both departments to coordinate efforts. Currently, there is an overlap between first responder and military operations, as was seen during the terrorist attacks of September 11, 2001 and the Hurricane Katrina disaster. Both of these events discovered major discontinuities between military communications systems and first responder networks. By coordinating the GIG and NG9-1-1 initiatives, better networks will be developed, which means more lives saved for both the military and first responders.

The three key points raised here (architecture, applications, and interoperability) form the basis of the synergies that can be developed between DoD and DoT during the development of their respective initiatives. Time, money, and manpower can be saved with cooperation between the departments.

There is a common thread to both initiatives in the architecture, which is the availability of information anywhere, any time. The architectures are interchangeable in some instances, and these instances can be leveraged to improve services to both first responders and war fighters alike. Applications are similar in most instances in the GIG and NG9-1-1 networks. In addition, the environments in which they will be used are comparable, from the individual first responder to the battlefield commander. Finally, interoperability and the problems posed by it between the users, the network, and the applications are a requirement in both networks.

After analyzing the many similarities within each initiative, the question of who and how cooperation can be fostered needs to be answered. The obvious answer as to who could foster cooperation should be the DHS. Not only is the DHS involved in military planning to some extent, but also they are also extremely interested in all facets of first responder operations. With the DHS's strong science and technology capability, they have the resources and ability to provide the bridge that can draw these two initiatives together.

\section{REFERENCES}

[1] U.S. Department of Transportation, Next Generation 9-1-1 (NG9-1-1) System Initiative Concept of Operations, Version 2.0. April 6, 2007.

[2] U.S. Department of Transportation, Next Generation 9-1-1 (NG9-1-1) System Initiative System Description and High-Level Requirements Document, Version 1.1. July $31,2007$.

[3] U.S. Department of Defense, Department of Defense Global Information Grid Architectural Vision, Version 1.0. June 1997. 